\title{
Reovirus as a novel oncolytic agent
}

\author{
Kara L. Norman and Patrick W.K. Lee \\ Department of Microbiology and Infectious Diseases, University of Calgary, Calgary, Canada \\ Address correspondence to: Patrick W.K. Lee, Department of Microbiology and Infectious Diseases, Room B855, \\ Health Sciences Building, University of Calgary, 3330 Hospital Drive NW, Calgary, Alberta T2N 2N1, Canada. \\ Phone: (403) 220-7548; Fax: (403) 270-8520; E-mail: plee@ucalgary.ca.
}

Reovirus is a double-stranded RNA-containing virus that possesses the distinctive ability to replicate in transformed cells while sparing normal cells, both in vitro and in vivo, in rodent models of cancer. The discovery of this property only arose through years of basic research on the biology of reovirus infection. Upon elucidation of the intracellular factors that govern cellular susceptibility, it became clear that reovirus Type 3 Dearing was capable of replicating in cells with an activated Ras signaling pathway, whereas normal, untransformed cells were unable to support reovirus infection (1). Because normal cells are resistant to reovirus, it is not surprising that reovirus infection in humans is usually subclinical $(2,3)$. Altogether, the potential impact of such findings is impressive when one considers that activating mutations in the ras genes alone contribute to more than $30 \%$ of all human cancers and that many other mutations in elements of the Ras pathway can also contribute to oncogenesis $(4,5)$. Recently, research using murine cancer models has revealed that this genetically unmodified laboratory strain of reovirus can indeed selectively destroy tumor cells, with no manifestations of animal morbidity or mortality (6). This has led the way to investigation into its therapeutic potential in human cancer.

\section{Background}

Reovirus belongs to the Reoviridae family, which includes viruses of a variety of tropisms (with examples of animal-, plant-, and insect-pathogenic viruses) and, most notably, the major human pathogen, rotavirus (7). Unlike the clinically significant rotavirus, reovirus is relatively nonpathogenic in humans. In 1959, Sabin coined the name reovirus as a descriptive acronym signifying its nonpathogenicity (8). This acronym refers to the fact that although reovirus can be isolated from the respiratory and enteric tracts, there is a lack of association of infection with clinical symptoms and it is, hence, an orphan virus. There are three serotypes of reovirus, based on their hemagglutinationinhibition activity. Prototypical laboratory strains of each serotype were isolated from children's respiratory and enteric tracts and are designated Type 1 Lang, Type 2 Jones, Type 3 Abney, and Type 3 Dearing.

All three serotypes of reovirus are found ubiquitously in the environment, including such sources as water and sewage. This, combined with the fact that reovirus possesses a highly stable unenveloped icosahedral capsid, explains why as many as $50 \%$ of adults aged $20-30$ years have been exposed to reovirus over the course of their lives and thus carry antibodies against the virus (9). Seropositivity has been documented to be as high as $70-100 \%$ of subjects in some studies $(10,11)$, despite the fact that most reovirus infections go unnoticed. In 1963, volunteers from a correctional institution demonstrated the nonpathogenic nature of reovirus; upon intranasal inoculation by serotypes 1,2 , or 3 , only nine of 27 subjects developed symptoms (3). Symptoms were uniformly mild and ranged from sneezing to pharyngitis. Moreover, serologic and virologic tests indicated that not all that fell ill were productively infected with reovirus but that some subjects with no symptoms were seropositive, confirming that infection leading to immunity is often benign.

\section{Selective oncolysis by reovirus}

The perception that reovirus is relatively nonpathogenic initially defined reovirus biology as chiefly a valuable model of viral replication and pathogenesis. Reovirus's potential cancer connection began in 1977, when Hashiro et al. documented the susceptibility of transformed cells to reovirus replication and also found that "normal," untransformed cells were resistant to the virus (12). Later, Duncan et al. (13) showed that WI-38 cells not normally susceptible to reovirus could be rendered infectible upon transformation by the SV-40 large Tantigen. Although these studies hinted at the oncolytic potential of reovirus, the mechanism behind cellular susceptibility to reovirus remained equivocal.

The mechanism by which reovirus causes tumor cell lysis came to light serendipitously during reovirus receptor studies. These studies revealed that although the receptor for viral attachment was the ubiquitous sialic acid, not all cells bearing this moiety supported a productive infection by reovirus. Furthermore, cells with high levels of EGF-R sustained viral replication, compared with those with low levels of the receptor (14). These observations, together with the demonstration that reovirus can bind directly to the EGF-R, suggest that the receptor either served as a site of viral attachment and entry or effected a change in the intracellular environment that conferred susceptibility. Evidence for the latter possibility came from studying NIH-3T3 fibroblasts transformed with the oncogene v-erbB, which encodes a constitutively activated receptor lacking the extracellular domain. Untransformed NIH-3T3 cells were not infectible, but v-erbB-transformed cells enabled efficient replication of reovirus, demonstrating that constitutive tyrosine kinase activity of the EGF-R, but not the extracellular domain of the receptor, was required for viral replication (15).

Tyrosine kinase activity of the EGF-R is normally stimulated in response to ligand binding to the receptor's extracellular domain (16). This in turn leads to autophos- 


\section{Figure 1}

Usurpation of the Ras signaling pathway by reovirus. In untransformed, reovirus-resistant cells, double-stranded RNA structures in reovirus transcripts activate PKR, which subsequently phosphorylates elF- $2 \alpha$, inhibiting translation initiation of viral genes. In cells with an activated Ras signaling pathway, however, PKR phosphorylation in response to viral transcripts is inhibited and viral translation proceeds unimpeded.

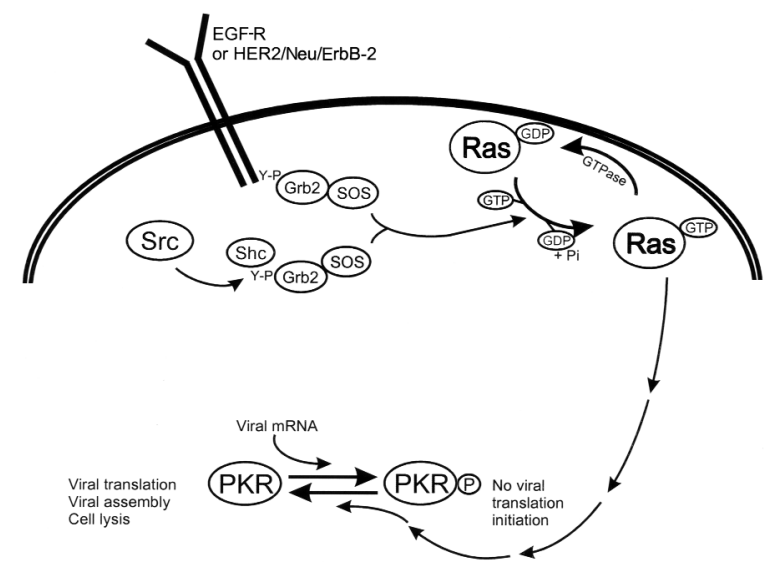

PKR in the antiviral response of normal cells (1).

Exactly how Ras and elements upstream of Ras signal to inhibit PKR's antiviral response is currently under investigation. Several years ago, Mundschau and Faller isolated a Ras-inducible PKR kinase inhibitor, termed RIKI (20, 21). RIKI appears to prevent PKR's activity through dephosphorylation of the enzyme, but a direct interaction between RIKI and PKR has not been shown. Many pathways downstream of Ras may impinge on the inhibitor of PKR. Activation of the MAPK pathway has been shown to correlate well with reovirus susceptibility; however, this relationship is not absolute. Furthermore, inhibition of this pathway with the MAPK kinase inhibitor, PD98059, does not alter PKR inhibition in $\mathrm{H}$ ras-transformed cells (1). Studies are ongoing to determine whether other pathways downstream of Ras, for example the SAPK/JNK cascade or the p38 stress-activated kinase cascade, may impinge on PKR and/or are involved in reovirus infection.

\section{Animal tumor models}

Once it was established that Ras pathway activation determined reovirus susceptibility in cell culture and that activation of this pathway was also important in human cancer, reovirus emerged as a plausible oncolytic agent (6). The first experiments conducted involved testing reovirus on a variety of human cancer cell lines in vitro. So far, more than $80 \%$ of human tumor cell lines of various origins have proved to be infectible (our unpublished data). Following upon this success against human cancer cell lines in vitro, reovirus was tested in vivo for its capacity to kill tumor cells and leave normal tissue architecture intact. We first tested the oncolytic capacity of reovirus in tumor xenografts in a severe combined immunodeficient (SCID) mouse model in which v-erbB-transformed NIH-3T3 fibroblasts were implanted subcutaneously above the hind flank. Once palpable tumors had been established, mice were administered a single intratumoral injection. After a period of 12 days, the tumors had regressed significantly in six of eight mice. Similarly, using the human glioblastoma cell line U87, tumors regressed in four of five mice after 4 weeks. The use of other human cancer cell lines as tumor xenografts (including breast, prostate, and colorectal 
cancer) yielded similar results (our unpublished data).

We proceeded to test reovirus in immunocompetent animals to determine whether tumor regression could occur in the presence of a potentially therapy-inactivating immune response (6). In this model, we implanted C3H 10T1/2 fibroblasts (transformed with activated ras) subcutaneously into $\mathrm{C} 3 \mathrm{H}$ mice and treated the tumor allografts with multiple intratumoral injections of reovirus over a course of 21 days. In the presence of a competent immune system, reovirus still caused complete tumor regression in $80 \%$ of the animals. Although more virus was required for a complete response, no side effects were noted and no tumor regrowth occurred for a period of 6 months after eradication of the tumor mass. Furthermore, whereas tumor cells treated with ultraviolet-inactivated virus infiltrated surrounding muscle tissue, live-virus-treated tumors were completely cleared. Virus was not present in any of the normal tissues examined. As most individuals have had previous exposure to the virus and thus carry neutralizing antibodies that could inactivate the therapy, another study was undertaken to reflect more accurately the typical seropositivity of humans. It was found that prior exposure of mice to the virus, causing an immune response, did not affect the ability of reovirus to kill the tumor. The combined data from these studies indicate that reovirus was capable of eradicating tumors in vivo, in both an immunodeficient and an immunocompetent environment.

\section{Future directions}

Further investigation is now warranted into the effectiveness of reovirus as a systemic treatment of metastases and as an adjuvant therapy in combination with chemotherapeutics. Emerging data using a SCID mouse model indicate that reovirus can spread systemically to cause regression in tumors remote from the site of injection (our unpublished data), which could prove useful in the treatment of metastases, and also raises the possibility that the route of injection could be altered to that of an intravenous delivery. Intravenous injection may allow reovirus to reach and abolish inaccessible tumors and to treat undetectable micrometastases, but it might also affect the therapy's effectiveness and toxicity.

Reovirus-based cancer treatment, concurrent with chemotherapeutics, has not been investigated with respect to its role as strictly an oncolytic agent. However, reovirus has been assayed as an immunotherapy against cancer in conjunction with BCNU, a chemotherapeutic agent. Previous reports have shown that treatment of L1210 lymphoma ascites tumors concurrent with BCNU helps improve the survivability of mice, compared with untreated controls $(22,23)$. They postulate that reovirus potentiates immune responses to tumor antigens, noting that surviving mice reject subsequent challenge with homologous tumor. In contrast, in our hind flank $\mathrm{C} 3 \mathrm{H}$ tumor model, mice can in fact be successfully reimplanted with homologous tumor after initial cure with reovirus (our unpublished data), suggesting that immune responses were not stimulated against the allografts themselves, even though the animals mounted an immune response against reovirus proteins during treatment. Also of note, the immunosuppressive effects of cancer therapeutics may influence the potential efficacy and toxicity of reovirus treatment. Studies are needed to explain the interaction of the therapy with the immune system and to reconcile these conflicting observations described here.

Reovirus interactions with the Ras signaling pathway take on relevance when one considers the importance of this pathway clinically in cancer initiation and progression. In fact, greater than $30 \%$ of all cancers have activating mutations in the $\mathrm{H}-, \mathrm{N}-$, or K-ras genes, typically occurring at amino acids 12,13 , or 61 and leading to the accumulation of the active, GTP-bound form of Ras (16). Ras-GTP is capable of stimulating several cellular signal transduction pathways such as those mentioned here. The ras gene is frequently mutated in colorectal cancer (40-50\%); lung adenocarcinomas; and acute myelogenous, chronic myelomonocytic, and acute lymphoblastic leukemias (24-26). Pancreatic carcinomas have as much as a $95 \%$ occurrence of ras mutation $(4,27)$.

Neoplasms also may carry mutations in other genes, such as those for receptor and nonreceptor tyrosine kinases, that lead to constitutive Ras activity. Thus, cells with an activated c-erbB gene, encoding the EGF$\mathrm{R}$, are infectible by reovirus. Importantly, $38-50 \%$ of glioblastomas show amplification of c-erbB $(28,29)$. Its homologue, c-erbB-2/HER2/neu is frequently amplified in breast and ovarian carcinomas and is a particularly poor prognostic indicator of the disease $(30,31)$. To date, cells transformed with neu have been found to be infectible by reovirus, as are a high proportion of breast cancer cell lines. Breast cancers have also been documented to have high levels of nonreceptor tyrosine kinase activity, mostly due to the Src family of nonreceptor tyrosine kinases (32). High activity of Src can activate Ras pathway members and can render a cell infectible by reovirus. Finally, the Bcr-Abl fusion protein, a clinically relevant nonreceptor tyrosine kinase that is present in $95 \%$ of chronic myelogenous leukemias, depends on Ras activation to effect cellular transformation. Hence, neoplasms expressing Bcr-Abl, including those found in carriers of the Philadelphia chromosome (33), could also be potential targets for reovirus treatment.

\section{Conclusions}

Activation of the Ras signaling pathway, both through direct mutation of the ras proto-oncogene itself and through genetic alteration of upstream pathway elements, profoundly affects neoplastic genesis and progression. Although this activation can deregulate cellular growth and serves as a key step in tumorigenesis, it also offers an opportunity for reovirus to replicate in a variety of tumor cells. Reovirus lends itself well to a role as a cancer biotherapeutic because of its relatively nonpathogenic nature; it does not replicate in untransformed cells and does not cause morbidity or mortality upon tumor therapy in immunocompetent murine cancer models. Although there remain some unanswered questions with regard to the exact biochemistry of infection and the effect of therapy dose and route alteration, the ultimate challenge of tumor treatment in the human organism should prove to be the most interesting. 


\section{Acknowledgments}

The authors thank D. Browman for careful review of the manuscript. This research was supported by a grant (to P.W.K. Lee) from the Medical Research Council of Canada. K.L. Norman is supported by studentships from the Natural Sciences and Engineering Research Council of Canada and the Alberta Heritage Foundation for Medical Research.

1. Strong, J.E., Coffey, M.C., Tang, D., Sabinin, P., and Lee, P.W. 1998. The molecular basis of viral oncolysis: usurpation of the Ras signaling pathway by reovirus. EMBO J. 17:3351-3362.

2. Rosen, L., Hovis, J., Mastrota, F.M., Bell, J.A., and Huebner, R.J. 1960 Observations on a newly recognized virus (Abney) of the reovirus family. American Journal of Hygiene. 71:258-265.

3. Rosen, L., Evans, H.E., and Spickard, A. 1963. Reovirus infections in human volunteers. American Journal of Hygiene. 77:29-37.

4. Bos, J.L. 1989. ras oncogenes in human cancer: a review [erratum 1990, 50:1352]. Cancer Res. 49:4682-4689.

5. Hanahan, D., and Weinberg, R.A. 2000. The hallmarks of cancer. Cell. 100:57-70.

6. Coffey, M.C., Strong, J.E., Forsyth, P.A., and Lee, P.W. 1998. Reovirus therapy of tumors with activated Ras pathway. Science. 282:1332-1334.

7. Tyler, K.L., and Fields, B.N. 1996. Reoviruses. In Fields virology. B.N Fields, D.M. Knipe, and P.M. Howley, editors. Lippincott-Raven. Philadelphia, Pennsylvania, USA. 1597-1623.

8. Sabin, A.B. 1959. Reoviruses: a new group of respiratory and enteric viruses formerly classified as ECHO type 10 is described. Science. 130:1387-1389.

9. Jackson, G.G., and Muldoon, R.L. 1973. Viruses causing common respiratory infection in man. IV. Reoviruses and adenoviruses. J. Infect. Dis. 128:811-833.

10. Minuk, G.Y., Paul, R.W., and Lee, P.W. 1985. The prevalence of antibodies to reovirus type 3 in adults with idiopathic cholestatic liver disease. J. Med. Virol. 16:55-60.

11. Minuk, G.Y., et al. 1987. Reovirus type 3 infection in patients with primary biliary cirrhosis and primary sclerosing cholangitis. J. Hepatol. 5:8-13.

12. Hashiro, G., Loh, P.C., and Yau, J.T. 1977. The preferential cytotoxicity of reovirus for certain transformed cell lines. Arch. Virol. 54:307-315.

13. Duncan, M.R., Stanish, S.M., and Cox, D.C. 1978. Differential sensitivity of normal and transformed human cells to reovirus infection. J. Virol. 28:444-449.

14. Strong, J.E., Tang, D., and Lee, P.W. 1993. Evidence that the epidermal growth factor receptor on host cells confers reovirus infection efficiency. Virology. 197:405-411.

15. Strong, J.E., and Lee, P.W. 1996. The v-erbB oncogene confers enhanced cellular susceptibility to reovirus infection. J. Virol. 70:612-616.
16. Campbell, S.L., Khosravi-Far, R., Rossman, K.L., Clark, G.J., and Der, C.J. 1998. Increasing complexity of Ras signaling. Oncogene. 17:1395-1413.

17. Bischoff, J.R., and Samuel, C.E. 1989. Mechanism of interferon action. Activation of the human P1/eIF-2 alpha protein kinase by individual reovirus s-class mRNAs: $\mathrm{s} 1 \mathrm{mRNA}$ is a potent activator relative to $\mathrm{s} 4$ mRNA. Virology. 172:106-115.

18. Thomis, D.C., and Samuel, C.E. 1993. Mechanism of interferon action: evidence for intermolecular autophosphorylation and autoactivation of the interferon-induced, RNA dependent protein kinase PKR. J. Virol. 67:7695-7700.

19. Panniers, R., and Henshaw, E.C. 1983. A GDP/GTP exchange factor essential for eukaryotic initiation factor 2 cycling in Ehrlich ascites tumor cells and its regulation by eukaryotic initiation factor 2 phosphorylation. J. Biol. Chem. 258:7928-7934.

20. Mundschau, L.J., and Faller, D.V. 1992. Oncogenic ras induces an inhibitor of double-stranded RNA-dependent eukaryotic initiation factor 2 alpha-kinase activation. J. Biol. Chem. 267:23092-23098.

21. Mundschau, L.J. and Faller, D.V. 1994. Endogenous inhibitors of the dsRNA-dependent eIF-2 alpha protein kinase PKR in normal and rastransformed cells. Biochimie. 76:792-800.

22. Williams, M.E., Cox, D.C., and Stevenson, J.R. 1986. Rejection of reovirus-treated L1210 leukemia cells by mice. Cancer Immunol. Immunother. 23:87-92.

23. Steele, T.A., and Cox, D.C. 1995. Reovirus type 3 chemoimmunotherapy of murine lymphoma is abrogated by cyclosporine. Cancer Biother. 10:307-315.

24. Forrester, K., Almoguera, C., Han, K., Grizzle, W.E., and Perucho, M. 1987. Detection of high incidence of K-ras oncogenes during human colon tumorigenesis. Nature. 327:298-303.

25. Bos, J.L., et al. 1987. Prevalence of ras gene mutations in human colorectal cancers. Nature. 327:293-297.

26. Beaupre, D.M., and Kurzrock, R. 1999. RAS and leukemia: from basic mechanisms to gene-directed therapy. J. Clin. Oncol. 17:1071-1079.

27. Almoguera, C., et al. 1988. Most human carcinomas of the exocrine pancreas contain mutant c-K-ras genes. Cell. 53:549-554.

28. Brison, O. 1993. Gene amplification and tumor progression. Biochim. Biophys. Acta. 1155:25-41.

29. Bredel, M., and Pollack, I.F. 1999. The p21-Ras signal transduction pathway and growth regulation in human high-grade gliomas. Brain Res. Brain Res. Rev. 29:232-249.

30. Slamon, D.J., et al. 1989. Studies of the HER-2/neu proto-oncogene in human breast and ovarian cancer. Science. 244:707-712.

31. Kim, H., and Muller, W.J. 1999. The role of the epidermal growth factor receptor family in mammary tumorigenesis and metastasis. Exp. Cell Res. 253:78-87.

32. Ottenhoff-Kalff, A.E., et al. 1992. Characterization of protein tyrosine kinases from human breast cancer: involvement of the c-src oncogene product. Cancer Res. 52:4773-4778.

33. Zou, X., and Calame, K. 1999. Signaling pathways activated by oncogenic forms of Abl tyrosine kinase. J. Biol. Chem. 274:18141-18144. 